\title{
Application of deep learning to the classification of uterine cervical squamous epithelial lesion from colposcopy images combined with HPV types
}

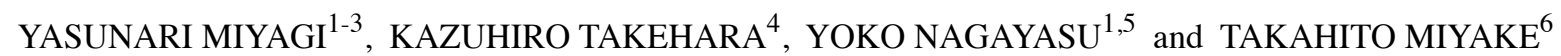 \\ ${ }^{1}$ Medical Data Labo, Okayama, Okayama 703-8267; ${ }^{2}$ Department of Gynecologic Oncology, Saitama Medical University \\ International Medical Center, Hidaka, Saitama 350-1298; ${ }^{3}$ Miyake Ofuku Clinic, Okayama, Okayama 701-0204; \\ ${ }^{4}$ Department of Gynecologic Oncology, National Hospital Organization Shikoku Cancer Center, Matsuyama, \\ Ehime 791-0208; ${ }^{5}$ Department of Obstetrics and Gynecology, Osaka Medical College, Takatsuki, Osaka 569-0801; \\ ${ }^{6}$ Department of Obstetrics and Gynecology, Miyake Clinic, Okayama, Okayama 701-0204, Japan
}

Received July 22, 2019; Accepted October 10, 2019

DOI: $10.3892 / 01.2019 .11214$

\begin{abstract}
The aim of the present study was to explore the feasibility of using deep learning, such as artificial intelligence (AI), to classify cervical squamous epithelial lesions (SILs) from colposcopy images combined with human papilloma virus (HPV) types. Among 330 patients who underwent colposcopy and biopsy performed by gynecological oncologists, a total of 253 patients with confirmed HPV typing tests were enrolled in the present study. Of these patients, 210 were diagnosed with high-grade SIL (HSIL) and 43 were diagnosed with low-grade SIL (LSIL). An original AI classifier with a convolutional neural network catenating with an HPV tensor was developed and trained. The accuracy of the AI classifier and gynecological oncologists was 0.941 and 0.843 , respectively. The AI classifier performed better compared with the oncologists, although not significantly. The sensitivity, specificity, positive predictive value, negative predictive value, Youden's J index and the area under the receiver-operating characteristic curve \pm standard error for AI colposcopy combined with HPV types and pathological results were $0.956(43 / 45), 0.833(5 / 6)$, 0.977 (43/44), 0.714 (5/7), 0.789 and $0.963 \pm 0.026$, respectively. Although further study is required, the clinical use of AI for the classification of HSIL/LSIL by both colposcopy and HPV type may be feasible.
\end{abstract}

\section{Introduction}

Recently, artificial intelligence (AI) has made remarkable progress in medicine. Humanity will undergo a dramatic and irreversible change when AI becomes very advanced, which

Correspondence to: Professor Yasunari Miyagi, Medical Data Labo, 289-48 Yamasaki, Naka, Okayama, Okayama 703-8267, Japan E-mail: ymiyagi@mac.com

Key words: colposcopy, HPV, cervical intraepithelial neoplasia, deep learning, artificial intelligence will likely occur in this century (1). AI has exceeded human experts in the field of games with perfect results (2), revealing novel strategies or findings. Therefore, as AI may be able to recognize certain information that conventional procedures cannot, it may also provide more precise diagnosis in practical medicine. Additionally, AI may be able to assist clinicians in practical medicine, reducing time and effort. For example, it has been reported that using AI-assisted colposcopy may reduce the time and effort it takes for a gynecologist to become a colposcopy expert, resulting in more time to improve other skills, training and activities (3). Moreover, the use of AI for predicting live births from blastocysts, to a level similar to that of specialists, may result in time saved for embryologists, reducing the financial costs of training (4). The aim of the present study was to investigate the feasibility of applying deep learning, a type of AI using both image and non-image information simultaneously, for gynecological clinical practice.

Uterine cervical cancer is a major public health problem as it is the third most common cancer in women and the leading cause of cancer-associated mortality among women in Central America, South-Central Asia, Middle and Western Africa and Melanesia (5). New methodologies to prevent cervical cancer should be made available and accessible to women in all countries (5).

Colposcopy is a well-established procedure for examining the uterine cervix under magnification (6-8). When lesions are treated with $3-5 \%$ acetic acid, colposcopy can detect and recognize cervical intraepithelial neoplasia (CIN) (6). Classification systems, such as the Bethesda system established in 2002, are used to categorize lesions as low-grade squamous intraepithelial lesions (LSILs) or high-grade SILs (HSILs) $(9,10)$, previously referred to as CIN1 and CIN2/CIN3, respectively (9). In clinical practice, distinguishing HSIL from LSIL in biopsy specimens is important as further examination or treatment, such as conization, may be required for HSIL.

In 2003, Burd (11) revealed that the Human papilloma virus (HPV) is essential to the transformation of the cervical epithelium. Based on genomic differences, DNA sequencing has identified $>200$ types of HPV, which can be grouped into 
low-risk (including types 6, 11, 42, 43 and 44) and high-risk (including types 16, 18, 31, 33, 34, 35, 39, 45, 51, 52, 56, 58, 59, 66, 68 and 70) HPV. In the high-risk group, certain HPV types are less frequently identified in cancers but are often present in SIL cells. The risk of progression for HPV types 16 and 18 is greater by $\sim 40 \%$ compared with that for other HPV types (11). Thus, HPV types may be associated with SILs because high-risk HPV may be more detectable in HSILs compared with LSILs. Information on HPV types may be beneficial to SIL diagnosis. However, the possibility of combining colposcopy findings with HPV types has not been previously explored.

Deep learning with a convolutional neural network $(12,13)$ to the realm of AI was applied to develop an original classifier for predicting HSIL or LSIL from colposcopy images (3) and HPV types. The aim of the present study was to determine whether AI could accurately evaluate colposcopy findings (combined with HPV types), compared with conventional colposcopy findings by gynecologic oncologists, and also to investigate the feasibility of applying deep learning (a class of AI using both image and non-image information simultaneously) in clinical gynecological clinical practice.

\section{Materials and methods}

Patients. The present study used fully de-identified patient data and was approved by the Institutional Review Board of Shikoku Cancer Center (approval no. 2017-81). The study was explained to patients who were not limited by age, had no prior treatment of the uterine cervix and had advanced lesions of the cervix biopsied at Shikoku Cancer Center between January 2012 and December 2017. Patients were directed to a website with additional information, including an opt-out option for the study. As the present study was a fully de-identified retrospective study, the Institutional Review Board of Shikoku Cancer Center approved the informed consent by the explanation including the opt-out option for patients to choose to withdraw from this study as informed consent. HPV tests had been performed in routine examination for patients with abnormal cervical cytology reports or abnormal colposcopy findings indicating neoplastic disease diagnosed by gynecological oncologists at Shikoku Cancer Center. HPV tests were not performed specifically for this study. Gynecological oncologists determined the necessity for biopsy in routine conventional practice for patients with abnormal cervical cytology reports of ASC-US, LSIL, HSIL, atypical squamous cells cannot ruled out HSIL (ASC-H), squamous cell carcinoma (SCC), adenocarcinoma in situ (AIS), atypical glandular cells (AGC) and adenocarcinoma (Adenoca), as well as for patients with abnormal cervical cytology reports or abnormal colposcopy findings indicating neoplastic disease such as LSIL/CIN1, HSIL/CIN2 and HSIL/CIN3. HPV types were tested by either one of the following commercially available PCR-based assay kits: Cobas ${ }^{\circledR} 4800$ system HPV (Roche Diagnostics), which detects high-risk HPV genotypes such as 16, 18, 31, 33, 35, 39, 45, 51, 52, 56, 58, 59, 66 and 68; or Amplicor ${ }^{\circledR}$ HPV (Roche Diagnostics), which detects high-risk HPV genotypes such as 16, $18,31,33,35,39,45,51,52,56,58,59$ and 68 . The design of the study was based on the routine practice at Shikoku Cancer Center. A total of 253 patients who underwent cervical punch biopsy combined with HPV typing test and had an image of colposcopy captured were enrolled in this study.
Images. Colposcopy images of lesions processed with 3\% acetic acid prior to biopsy were captured, cropped and saved in JPEG format. The image data were input retrospectively for deep learning.

AI preparation. All de-identified images saved offline were transferred to the AI system. For the test dataset, $20 \%$ of the images were randomly selected; the remaining images were used as the training dataset. Next, $80 \%$ of the training dataset images were used to train the AI classifier, and the remaining images were used as the validation dataset. Thus, these datasets did not overlap. The AI classifier was trained using a training dataset and simultaneously validated and tested using the test dataset. The training datasets were augmented, because colposcopy image processing of arbitrary degrees of rotation can yield images resulting in different vector data for the same category.

AI classifier. Classifier programs were developed using supervised deep learning with a convolutional neural network architecture $(12,14)$ catenated with a HPV type tensor. A number of convolutional neural networks were tested by varying image size (50x50, 75x75 and 100x100 pixels), L2 regularization $(15,16)$ and architectures consisting of a combination of convolution layers with kernels (17-19), pooling layers (20-23), flattened layers (24), linear layers $(25,26)$, rectified linear unit layers $(27,28)$, catenated layers, batch normalization layers (29) and a softmax layer $(30,31)$ which demonstrated the probability of LSIL or HSIL from an image (Table I).

Cross-validation (32-34), which is a method for model selection, was applied to identify the optimal machine learning method. The suitable number of images for the training data was determined using the 5-fold cross-validation method, which reveals the optimal number of training data and can be used to avoid overfitting, a modeling error that occurs when a classifier is too closely fit to a limited set of data points (35-40). After the optimal number of training data was calculated, the classifier that exhibited the highest accuracy was selected. Conventional colposcopy diagnosis and AI colposcopy diagnosis-catenated HPV types in the test dataset were compared. A flow chart of the development of the AI classifier is presented in Fig. 1.

Development environment. The following development environment was used: A Macintosh running OS X 10.14.5 (Apple, Inc.) and Mathematica 12.0.0.0 (Wolfram Research, Inc.).

Statistical analysis. The laboratory and AI classifier data were compared using Mathematica 12.0.0.0 (Wolfram Research, Inc.). The Cochran Armitage test, Cohen's $\kappa, \chi^{2}$ test and Fisher's exact test were used. $\mathrm{P}<0.05$ was considered to indicate a statistically significant difference.

\section{Results}

The number of patients with cytology reports were stratified as follows: HSIL, 149; LSIL, 75; AUC-US, 43; ASC-H, 38; SCC, 18; AGC, 2; AIS; 2; Adenoca, 2; NILM, 8. Mean \pm standard deviation, median and range of patient age in the HSIL 
Table I. Architecture of the classifier.

\begin{tabular}{lc}
\hline Input Image & Input HPV Type \\
\hline 1. Convolutional layer & - \\
2. Rectified linear unit layer & - \\
3. Pooling layer & - \\
4. Convolutional layer & - \\
5. Rectified linear unit layer & - \\
6. Pooling layer & - \\
7. Flattening layer & - \\
8. Linear layer & - \\
9. Rectified linear unit layer & \\
10. Linear layer & \\
Catenated layer & \\
Batch normalization layer & \\
Linear layer & \\
Softmax layer & \\
Output &
\end{tabular}

The classifier consisted of a combination of 10 layers of a convolutional neural network and a single layer of an HPV type tensor. The image processing and HPV type tensor were combined at the catenated layer. '-', no HPV type combined with the layer; HPV, human papilloma virus.

vs. LSIL groups were $31.66 \pm 5.01$ vs. $33.75 \pm 8.94,32$ vs. 33 and $19-46$ vs. 19-62, respectively. The pathological diagnoses and the corresponding number of patients who underwent punch biopsy were as follows: HSIL, 213; LSIL, 97; squamous cell carcinoma, 12; adenocarcinoma, 5; adenocarcinoma in situ, 2; and microinvasive squamous cell carcinoma, 1 (Table II). The HPV types of the patients were as follows: Type 16, 87; type 18,8 ; type 16 and 18,4 ; high-risk HPV but not type 16 or 18, 159; and HPV negative, 13. A total of 57 patients $(17.2 \%)$ did not receive a HPV type test. Conventional colposcopy diagnoses based on pathological results and HPV types are presented in Tables III and IV. A total of 20/273 patients had no image data.

A total of 253 patients with colposcopy images, pathological LSIL or HSIL and known HPV types were finally enrolled in this study. The ages of patients with pathological HSIL and LSIL were $31.66 \pm 4.83$ and $30.12 \pm 5.10$ (mean \pm standard deviation), respectively (data not shown). The median age (range) of pathological HSIL and LSIL were 32 (19-46) and 33 (19-62), respectively (data not shown). HPV types were reclassified as follows: Type 16 or 18 were considered high-risk HPV, and not type 16 or 18 was considered to represent low-risk HPV/HPV-negative due to the limited number of available HPV types and pathological results. HPV-negative described the absence of high-risk HPV, not type 16 or 18 .

The numbers of patients with type 16 and/or 18, high-risk HPV but not type 16 or 18 and HPV-negative were 85, 156 and 12, respectively (Table II). The numbers of patients with HSIL and LSIL were 210 and 43, respectively. Among the 210 pathological HSIL cases, the numbers of type 16 and/or 18 , high-risk HPV but not type 16 or 18 and HPV-negative were 81, 123 and 6, respectively. Among the 43 pathological LSIL cases, the numbers of type 16 and/or 18, high-risk HPV but not type 16 or 18 and HPV-negative were 4, 33 and 6, respectively. The HPV types were associated with the pathological results $\left(\mathrm{P}<6.70 \times 10^{-6}\right.$; Cochran Armitage test). HPV-negative results were observed in $2.85 \%$ (6/210) and $14.00 \%$ (6/43) of HSIL and LSIL, respectively. In the present study, type 16- or 18-positive HPV in pathological HSIL and LSIL were observed in 38.6 and $9.3 \%$ of cases, respectively. The incidence of type 16 and/or 18 positivity in pathological HSIL was significantly higher, compared with that in LSIL $(\mathrm{P}<0.0005$; Fisher's exact test with Yates's correction).

Among the 210 pathological HSIL cases, 177 patients received a conventional colposcopy diagnosis by gynecologists of CIN2 (HSIL) or CIN3 (HSIL), 29 were diagnosed with CIN1 (LSIL), three were diagnosed with invasive cancer and one was diagnosed with cervicitis. Among the 43 pathological LSIL cases, 13 received a conventional colposcopy diagnosis by gynecologists of HSIL, 25 were diagnosed with LSIL and 5 with cervicitis. The accurate diagnoses of HSIL and LSIL were 202 out of 253 (0.798). The accuracy, sensitivity, specificity, positive predictive value, negative predictive value and Youden's $\mathrm{J}$ index of the conventional colposcopy diagnosis for pathological HSIL were 0.828 (202/244), 0.859 (177/206), $0.658(25 / 38), 0.932(177 / 190), 0.463(25 / 54)$ and 0.517 , respectively.

Among the 85 cases with HPV type 16 and/or 18, 71 received a conventional colposcopy diagnosis by gynecologists of CIN2 (HSIL) or CIN3 (HSIL), 10 were diagnosed with CIN1 (LSIL), two with cervicitis and two with invasive cancer. Among the 156 cases with HPV type 16 and/or 18, 113, 40, 2 and 1 received a conventional colposcopy diagnosis by gynecologists of CIN2 (HSIL) or CIN3 (HSIL), CIN1 (LSIL), cervicitis and invasive cancer, respectively. Among the 12 HPV-negative cases 6, 4 and 2 received a conventional colposcopy diagnosis by gynecologists of CIN2 (HSIL) or CIN3 (HSIL), CIN1 (LSIL) and cervicitis, respectively. There are no relationships between HPV types and colposcopy.

The highest accuracy for HSIL of the best AI classifier combined with HPV types for a test dataset was $0.941(48 / 51)$ when the number of the augmented training dataset was 1,212, the value of $\mathrm{L} 2$ regularization was 0.02 , and the image size was 50x50 pixels. The accuracy, sensitivity, specificity, positive predictive value, negative predictive value, Youden's J index (41), the area under the receiver operating characteristic curve (AUC) \pm standard error, the $95 \%$ confidence interval of the AUC and Cohen's k (42) coefficients of HSIL for the AI colposcopy combined with HPV types and pathological results are presented in Table V.

The comparison of the conventional colposcopy diagnosis by gynecological oncologists and the best AI classifier for the test dataset is presented in Table VI. As the AI classifier was not trained for cervicitis or invasive cancer, when the colposcopy diagnosis was limited to HSIL and LSIL by ignoring colposcopy diagnoses of cervicitis and invasive cancer, the Cohen's k coefficient of the colposcopy diagnosis and the AI classifier was 0.407 . The agreement of the two methods was moderate (43), but not significant $(\mathrm{P}=0.077)$.

The comparison of the conventional colposcopy diagnosis by gynecological oncologists and the pathological results for the test dataset is presented in Table VI. The accurate number 


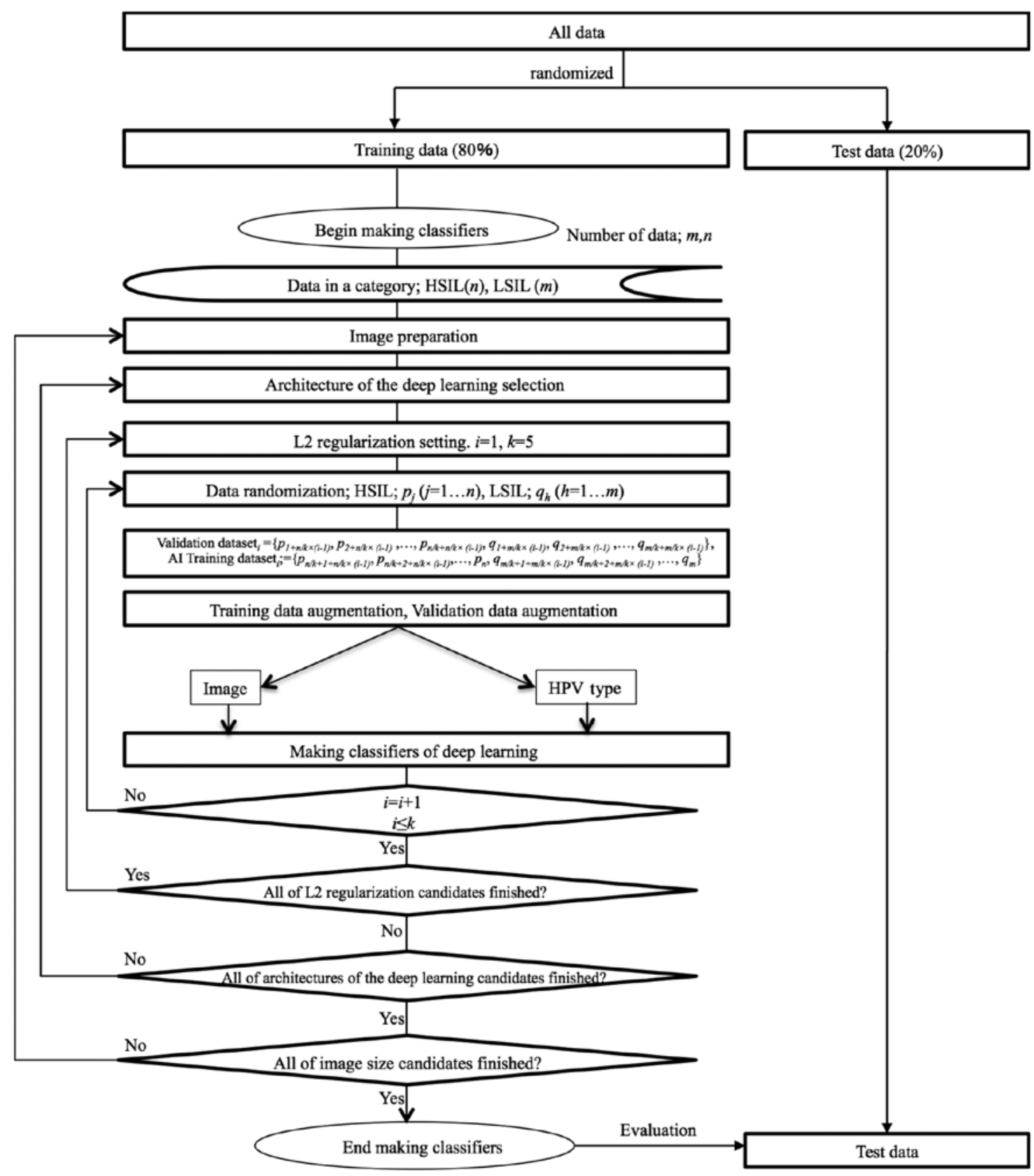

Figure 1. Development the AI classifier with deep learning for colposcopy images and HPV types. HPV, human papilloma virus; LSIL, low-grade squamous intraepithelial lesion; HSIL, high-grade squamous intraepithelial lesion; AI, artificial intelligence.

of HSIL and LSIL by conventional colposcopy for the test dataset was 43 of 51 (0.843). The conventional colposcopy results for the test dataset and for all of the datasets were not significantly different, and the time required for classification was $<0.2 \mathrm{sec}$ per patient.

\section{Discussion}

In the present study, a classifier was developed using deep learning with convolutional neural networks using images of cervical SILs combined with HPV types to predict the pathological diagnosis. The accuracy for the test dataset achieved by the classifier and by gynecological oncologists was 0.941 and 0.843 , respectively; the latter accuracy was calculated tentatively, and these two accuracies could not be compared as the AI was trained for HSIL and LSIL classes, whereas colposcopy could identify lesions such as cervicitis, invasive cancer and adenocarcinoma. The numbers of accurate HSIL and LSIL diagnoses by conventional colposcopy for the test dataset were 43 out of 51 and for all datasets were and 202 out of 253 . Compared with the classifier, the conventional colposcopy results for the test dataset and for all of the datasets were not significantly different, which suggested that the AI classifier using deep learning with convolutional neural networks using images of cervical SILs combined with HPV types was not inferior to conventional colposcopy performed by gynecologic oncologists.

In the present study, 12 cases of pathological HSIL and LSIL were HPV-negative, although both HPV type information and colposcopy images were used for analysis. These cases may have represented false negatives as HPV infection is essential to 
Table II. Patients with pathological results confirmed by punch biopsy and different HPV types.

Pathological results

\begin{tabular}{lrrrcrc} 
& & & Microinvasive & Invasive \\
HPV type & HSIL & LSIL & SCC & SCC & Adenocarcinoma in situ & Adenocarcinoma \\
\hline Not available & 3 & 54 & 0 & 0 & 0 & 0 \\
HPV-negative & 6 & 6 & 0 & 0 & 0 & 0 \\
High risk but not type 16 or 18 & 123 & 33 & 1 & 2 & 0 & 0 \\
Type 16 & 75 & 2 & 0 & 8 & 2 & 1 \\
Type 18 & 5 & 2 & 0 & 0 & 0 & 1 \\
Type 16+18 & 1 & 0 & 0 & 2 & 0
\end{tabular}

HPV, human papilloma virus; LSIL, low-grade squamous intraepithelial lesion; HSIL, high-grade squamous intraepithelial lesion; SCC, squamous cell carcinoma.

Table III. Patients with pathological results confirmed by punch biopsy and conventional colposcopy diagnosis by gynecologic oncologists.

\begin{tabular}{lccccc}
\hline & \multicolumn{4}{c}{ Colposcopy diagnosis } \\
\cline { 2 - 6 } Pathological results & CIN1 (LSIL) & CIN2 (HSIL) & CIN3 (HSIL) & Cervicitis & Invasive cancer \\
\hline HSIL & 32 & 63 & 114 & 1 & 3 \\
LSIL & 70 & 17 & 5 & 1 & 0 \\
Microinvasive SCC & 0 & 0 & 4 & 0 & 0 \\
Invasive SCC & 0 & 0 & 2 & 0 & 0 \\
Adenocarcinoma in situ & 0 & 0 & 1 & 0 & 0 \\
Adenocarcinoma & 0 & 0 & & 4 \\
\hline
\end{tabular}

LSIL, low-grade squamous intraepithelial lesion; HSIL, high-grade squamous intraepithelial lesion; SCC, squamous cell carcinoma; CIN, cervical intraepithelial neoplasia.

the transformation of cervical epithelial cells (11) and the HPV detection kits that were commercially available and widely used result in $<3.1 \%$ of false negatives in pathological HSIL in Japan as stated by the manufacturer. The data not excluded as only a small number of HPV-negative cases were identified. Previous studies have reported $8-13 \%$ of false negative results for HPV detection (44-46). Lee et al (47) reported that the classic nested PCR and Sanger DNA sequencing technology for routine HPV testing exhibited that a true negative HPV PCR invariably indicated the absence of precancerous cells in the cytology samples.

The accuracy of 0.941 was an acceptable result of the classifier for deep learning. In medicine, several studies have used AI for deep learning with convolutional neural networks $(48,49)$. The accuracy values of AI with deep learning have been published and include 0.997 for the histopathological diagnosis of breast cancer (50), 0.980 for the morphological quality of blastocysts and evaluation by an embryologist (51), 0.640-0.880 for predicting live birth from a blastocyst image of patients by age $(4,52), 0.650$ for predicting live birth without aneuploidy from a blastocyst image (53), 0.823 (3), 0.720 (54) and 0.500 (55) for colposcopy, 0.830 to 0.900 for the early diagnosis of Alzheimer's disease (56), 0.830 for urological dysfunctions (57) and 0.830 for the diagnostic imaging of orthopedic trauma (58). A number of studies have reported a limitation of conventional colposcopy. A study of the accuracy of biopsy under colposcopy reported a total biopsy failure rate, comprising both non-biopsy and incorrect selection of biopsy site, of 0.200 in CIN1, 0.110 in CIN2 and 0.090 in CIN3 (59). The colposcopic impression of high-grade CIN had a specificity of 0.880 and a sensitivity of 0.540 , as determined by nine expert colposcopists in 100 cervigrams (60). The sensitivity of an online colpophotographic assessment of HSIL by 20 colposcopists was 0.390 (61). Thus, conventional colposcopy does not provide good sensitivity, even when performed by colposcopy specialists. By contrast, the accuracy and sensitivity reported in this study for predicting HSIL from colposcopy images combined with HPV types using deep learning were 0.941 and 0.956 , respectively, which appears to be satisfactory. Since the classifier was not trained in colposcopy findings such as mosaic acetowhite epithelium and punctuation, it may recognize certain morphological features of cervical SILs by itself. It is also possible that the AI classifier may recognize features that colposcopists do not, such as relative or absolute brightness of acetowhite, complexity of the shape of the lesion, 
Table IV. Patients with all types of HPV and the conventional colposcopy diagnosis by gynecologic oncologists.

\begin{tabular}{lccccc}
\hline & \multicolumn{5}{c}{ Colposcopy diagnosis } \\
\cline { 2 - 6 } HPV type & CIN1 (LSIL) & CIN2 (HSIL) & CIN3 (HSIL) & Cervicitis & Invasive cancer \\
\hline Not available & 48 & 9 & 0 & 0 & 0 \\
HPV-negative & 4 & 5 & 1 & 2 & 1 \\
High risk but not type 16 or 18 & 40 & 46 & 70 & 2 & 1 \\
Type 16 & 9 & 18 & 50 & 1 & 9 \\
Type 18 & 1 & 2 & 5 & 1 & 1 \\
Type 16+18 & 0 & 0 & 1 & 0 & 3 \\
\hline
\end{tabular}

HPV, human papilloma virus; LSIL, low-grade squamous intraepithelial lesion; HSIL, high-grade squamous intraepithelial lesion; CIN, cervical intraepithelial neoplasia.

quantitative marginal evaluation of borders and distribution of punctuation density. The pathological results in the present study were obtained and defined by punch biopsy as it was not recommended for patients with CIN1 (LSIL) diagnosed by colposcopy to undergo conization or hysterectomy. The advanced lesion would have been revealed if the pathological results were defined by conization or hysterectomy rather than by punch biopsy; thus, both conventional colposcopy and the AI classifier may have demonstrated different results. When AI is used for advanced diseases, such as squamous cell carcinoma and adenocarcinoma, the pathological diagnosis should be provided by conization or hysterectomy.

It is important for clinicians to distinguish HSIL from LSIL in biopsy specimens in clinical practice as further examination or treatment, such as conization, may be required for HSIL. The clinician should consider biopsy when a reliable classifier indicates HSIL in clinical practice. The classifier developed in the present study may help untrained gynecologists avoid or reduce the risk of misidentifying HSIL. When the performance of the AI classifier is further improved in accuracy, sensitivity and specificity for classifying SILs, gynecologists may be able to obtain more precise classification without requiring a colposcopy specialist.

Several reasons for obtaining high accuracy by AI were considered in the present study. First, the association between the pathological results, colposcopy diagnosis and HPV types was important. The pathological results were affected by the HPV types. However, no association was identified between HPV types and the results of colposcopy. Thus, HPV types and colposcopy were associated with pathological results, but not with each other. In our preliminary study, the accuracy achieved by deep learning with only images of colposcopy was 0.823 (data not shown). Thus, the association among the pathological results, colposcopy diagnosis and HPV type may be a reason for high accuracy.

Second, AI has the ability to use images and non-image data simultaneously. However, AI is not established to digitize images to numerical data indicating the features of the images for multivariate analysis; AI, including deep learning, can acquire numerical data to indicate the features of an image and use the numerical data indicating the features of colposcopy images and the numeric tensor data of HPV types. This is an
Table V. The results of the best AI classifier combined with HPV types and conventional colposcopy for 51 test datasets (20\% of all qualified datasets).

\begin{tabular}{lcc}
\hline Variable & \multicolumn{1}{c}{ AI } & $\begin{array}{c}\text { Conventional } \\
\text { colposcopy }\end{array}$ \\
\hline Accuracy & $0.941(48 / 51)$ & $0.843(43 / 51)$ \\
Sensitivity & $0.956(43 / 45)$ & $0.844(38 / 45)$ \\
Specificity & $0.833(5 / 6)$ & $0.833(5 / 6)$ \\
Positive predictive value & $0.977(43 / 44)$ & $0.974(38 / 39)$ \\
Negative predictive value & $0.714(5 / 7)$ & $0.500(6 / 12)$ \\
Youden's J index & 0.789 & 0.677 \\
AUC \pm standard error $)$ & $0.963 \pm 0.026$ & N/A \\
Cohen's א & 0.769 & 0.473 \\
\hline
\end{tabular}

AUC, area under the receiver operating characteristic curve; HPV, human papilloma virus; AI, artificial intelligence.

important feature of AI, which may be the second reason for high accuracy in this study from the perspective of computer science.

Third, in the present study, the neural network architecture including a batch normalization layer (29) was adequate. Neural network architecture is a key component of deep learning. A batch normalization layer was added following catenating information from colposcopy images and HPV types. This method makes normalization a part of the model architecture and performs the normalization for each training mini-batch. Batch normalization allows the use of high learning rates. This architecture may be the third reason for high accuracy in the present study.

The architecture of the neural network has been progressing. The LeNet study in 1998 (62) consisted of 5 layers. AlexNet in 2012 (30) consisted of 14, and Google Net in 2014 (26) was constructed from a combination of micronetworks. ResNet-50 in 2015 (63) consisted of modules with a shortcut process. Squeeze-and-excitation networks were first published in 2017 (64). However, AI for image recognition remains 
Table VI. Comparison of the diagnosis of conventional colposcopy by gynecological oncologists and the best AI classifier and the pathological results for patients with HSIL or LSIL in the test dataset.

AI diagnosis (image combined with HPV type) Pathological diagnosis

\begin{tabular}{lcccc}
\cline { 2 - 4 } Colposcopy Diagnosis & HSIL & LSIL & HSIL & LSIL \\
\hline HSIL & 36 & 2 & 38 & 0 \\
LSIL & 6 & 4 & 5 & 5 \\
Cervicitis & 1 & 1 & 1 & 1 \\
Invasive cancer & 1 & 0 & 1 & 0
\end{tabular}

HPV, human papilloma virus; LSIL, low-grade squamous intraepithelial lesion; HSIL, high-grade squamous intraepithelial lesion; AI, artificial intelligence.

in development. Image information is one of the parameters requiring further investigation. Only $15 \times 15$ pixels have been used to detect cervical cancer (65); thus, image size remains an issue. In a previous colposcopy study, the reported accuracy for images of $150 \times 150$ pixels was higher compared with that for images of $300 \times 300$ or $32 \times 32$ pixels (55). In the present study, $111 \times 111,70 \times 70$ and 50x50 pixel images were tested. A size of 50x50 pixels, which exhibited the best performance in the present study, falls within the acceptable range. Regularization values that are routinely used in developing AI of deep learning are also an important hyperparameter for constructing a good classifier that avoids overfitting (35-40). Selecting the appropriate number of training datasets is also very important; in addition, the validation dataset prevents overfitting. Generally, more varied patterns of images may be needed for datasets as 500-1,000 images are reportedly prepared for each class during image classification with deep learning $(52,66)$. The classifier that uses both image and HPV types may require more images combined with HPV types, which may result in improvement in the classifier with deep learning.

In the present study, a classifier was developed based on deep learning that used both HPV types and images of uterine cervical SILs to predict pathological HSIL/LSIL. The accuracy of the classifier was 0.941. Although further study using more datasets and modified neural network architecture and/or hyperparameters is required to validate the classifier, the results of the present study demonstrated that AI may have a potential for clinical use in colposcopy examinations and may provide benefits to patients and clinicians.

\section{Acknowledgements}

Not applicable.

\section{Funding}

No funding was received.

\section{Availability of data and materials}

The datasets generated and analyzed during the current study are not publicly available since data sharing is not approved by the Institutional Review Board of Shikoku Cancer Center (approval no. 2017-81) but are available from the corresponding author on reasonable request.

\section{Author's contributions}

YM designed the study, programmed the AI, produced the AI classifiers, performed statistical analysis and wrote the manuscript. YM and YN designed the AI architecture. KT performed clinical intervention, data entry and collection, designed the study and critically reviewed the manuscript. YN critically reviewed the manuscript. TM designed the study and critically reviewed the manuscript.

\section{Ethics approval and consent to participate}

The protocol for this retrospective study used fully de-identified patient data and was approved by the Institutional Review Board of Shikoku Cancer Center (approval no. 2017-81). This study was explained to patients, who were also directed to a website with additional information, including an opt-out option that informed them of their right to not participate in this study. Written informed consent for this study design was not required, according to the guidance of the Ministry of Education, Culture, Sports, Science and Technology of Japan.

\section{Patient consent for publication}

Not applicable.

\section{Competing interests}

YM, YN and TM declare that they have no competing interests. KT declares receipt of personal funding from Taiho Pharmaceuticals, Chugai Pharma, AstraZeneca, Nippon Kayaku, Eisai, Ono Pharmaceutical, Terumo Corporation and Daiichi Sankyo.

\section{References}

1. Müller VC and Bostrom N (eds): Future progress in artificial intelligence: A survey of expert opinion. In: Fundamental Issues of Artificial Intelligence. Springer, Cham, pp555-572, 2016. 
2. Silver D, Schrittwieser J, Simonyan K, Antonoglou I, Huang A, Guez A, Hubert T, Baker L, Lai M, Bolton A, et al: Mastering the game of go without human knowledge. Nature 550: 354-359, 2017.

3. Miyagi Y, Takehara K and Miyake T: Application of deep learning to the classification of uterine cervical squamous epithelial lesion from colposcopy images. Mol Clin Oncol 11: 583-589, 2019.

4. Miyagi Y, Habara T, Hirata R and Hayashi N: Feasibility of predicting live birth by combining conventional embryo evaluation with artificial intelligence applied to a blastocyst image in patients classified by age. Reprod Med Biol 18: 344-356, 2019.

5. Arbyn M, Castellsagué X, de Sanjosé S, Bruni L, Saraiya M, Bray F and Ferlay J: Worldwide burden of cervical cancer in 2008. Ann Oncol 22: 2675-2686, 2011.

6. García-Arteaga JD, Kybic J and Li W: Automatic colposcopy video tissue classification using higher order entropy-based image registration. Comput Biol Med 41: 960-970, 2011

7. Kyrgiou M, Tsoumpou I, Vrekoussis T, Martin-Hirsch P, Arbyn M, Prendiville W, Mitrou S, Koliopoulos G, Dalkalitsis N, Stamatopoulos P and Paraskevaidis E: The up-to-date evidence on colposcopy practice and treatment of cervical intraepithelial neoplasia: The Cochrane colposcopy and cervical cytopathology collaborative group (C5 group) approach. Cancer Treat Rev 32: 516-523, 2006 .

8. O'Neill E, Reeves MF and Creinin MD: Baseline colposcopic findings in women entering studies on female vaginal products. Contraception 78: 162-166, 2008.

9. Waxman AG, Chelmow D, Darragh TM, Lawson H and Moscicki AB: Revised terminology for cervical histopathology and its implications for management of high-grade squamous intraepithelial lesions of the cervix. Obstet Gynecol 120: 1465-1471, 2012

10. Darragh TM, Colgan TJ, Thomas Cox J, Heller DS, Henry MR, Luff RD, McCalmont T, Nayar R, Palefsky JM, Stoler MH, et al: Members of the LAST project work groups. The lower anogenital squamous terminology standardization project for HPV-associated lesions: Background and consensus recommendations from the College of American Pathologists and the American society for colposcopy and cervical pathology. Int J Gynecol Pathol 32: 76-115, 2013.

11. Burd EM: Human papillomavirus and cervical cancer. Clin Microbiol Rev 16: 1-17, 2003.

12. Rumelhart D, Hinton G and Williams R: Learning representations by back-propagating errors. Nature 323: 533-536, 1986.

13. Bengio Y, Courville A and Vincent P: Representation learning: A review and new perspectives. IEEE Trans Pattern Anal Mach Intell 35: 1798-1828, 2013.

14. Schmidhuber J: Deep learning in neural networks: An overview. Neural Netw 61: 85-117, 2015.

15. Srivastava N, Hinton G, Krizhevsky A, Sutskever I and Salakhutdinov R: Dropout: A simple way to prevent neural networks from overfitting. J Mach Lean Res 15: 1929-1958, 2014

16. Nowlan SJ and Hinton GE: Simplifying neural networks by soft weight-sharing. Neural Comput 4: 473-493, 1992.

17. Bengio Y: Learning deep architectures for AI. Foundations and trends ${ }^{\circledR}$ in machine learning 2: 1-127, 2009.

18. Mutch J and Lowe DG: Object class recognition and localization using sparse features with limited receptive fields. Int J Comput Vision 80: 45-57, 2008

19. Neal RM: Connectionist learning of belief networks. Art Intell 56: 71-113, 1992.

20. Ciresan DC, Meier U, Masci J, Maria Gambardella L and Schmidhuber J: Flexible, high performance convolutional neural networks for image classification. IJCAI'11 Proceedings of the Twenty-Second international joint conference on artificial intelligence 2: 1237-1242, 2011

21. Scherer D, Müller A and Behnke S: Evaluation of pooling operations in convolutional architectures for object recognition In: Artificial Neural Networks-ICANN 2010. Diamantaras K, Duch W and Iliadis LS (eds). Lecture Notes in Computer Science Springer, Heidelberg, pp92-101, 2010.

22. Huang FJ and LeCun Y: Large-scale learning with SVM and convolutional for generic object categorization. Computer Vision and Pattern Recognition, 2006 IEEE Computer Society Conference 1: 284-291, 2006.

23. Jarrett $\mathrm{K}$, Kavukcuoglu $\mathrm{K}$, Ranzato $\mathrm{M}$ and LeCun $\mathrm{Y}$ : What is the best multi-stage architecture for object recognition? Computer vision. 12th IEEE international conference on computer vision, pp2146-2153, 2009.
24. Zheng Y, Liu Q, Chen E, Ge Y and Zhao JL: Time series classification using multi-channels deep convolutional neural networks. In: Web-Age Information Management. WAIM 2014. Lecture notes in computer science. Li F, Li G, Hwang S, Yao B and Zhang Z (eds). Springer, Cham, pp298-310, 2014

25. Mnih V, Kavukcuoglu K, Silver D, Rusu AA, Veness J, Bellemare MG, Graves A, Riedmiller M, Fidjeland AK, Ostrovski $\mathrm{G}$ et al: Human-level control through deep reinforcement learning. Nature 518: 529-533, 2015.

26. Szegedy C, Liu W, Jia Y, Sermanet P, Reed S, Anguelov D, Erhan D, Vanhoucke V and Rabinovich A: Going deeper with convolutions. Proceedings of the IEEE conference on computer vision and pattern recognition, pp1-9, 2015

27. Glorot X, Bordes A and Bengio Y: Deep sparse rectifier neural networks. Proceedings of the fourteenth international conference on artificial intelligence and statistics PMLR 15: 315-323, 2011.

28. Nair V and Hinton G: Rectified linear units improve restricted Boltzmann machines. Proceedings of the 27th International Conference on Machine Learning Haifa, pp807-814, 2010.

29. Ioffe S and Szegedy C: Batch Normalization: Accelerating Deep Network Training by Reducing Internal Covariate Shift. 32nd International Conference on Machine Learning, Lille, 2015.

30. Krizhevsky A, Sutskever I and Hinton GE: ImageNet Classification with Deep Convolutional Neural Networks. 25th International Conference on Neural Information Processing Systems, pp1097-1105, 2012.

31. Bridle JS: Probabilistic interpretation of feedforward classification network outputs, with relationships to statistical pattern recognition. In: Neurocomputing. Soulié FF and Hérault J (eds). Springer, Berlin, pp227-236, 1990.

32. Kohavi R: A study of cross-validation and bootstrap for accuracy estimation and model selection. Proceedings of the 14th international joint conference on artificial intelligence 2: 1137-1143, 1195.

33. Schaffer C: Selecting a classification method by cross-validation. Mach Lear 13: 135-143, 1993.

34. Refaeilzadeh $\mathrm{P}$, Tang $\mathrm{L}$ and Liu H: Cross-Validation. In: Encyclopedia of Database Systems. Liu L and Özsu MT (eds). Springer, Boston, pp532-538, 2009.

35. Yu L, Chen H, Dou Q, Qin J and Heng PA: Automated melanoma recognition in dermoscopy images via very deep residual networks. IEEE Trans Med Imaging 36: 994-1004, 2017.

36. Caruana R, Lawrence S and Giles CL: Overfitting in neural nets: Backpropagation, conjugate gradient, and early stopping. Advances in neural information processing systems 13: 402-408, 2001.

37. Baum EB and Haussler D: What size net gives valid generalization? Neural Computation 1: 151-160, 1989.

38. Geman S, Bienenstock E and Doursat R: Neural networks and the bias/variance dilemma. Neural Computation 4: 1-58, 1992.

39. Krogh A and Hertz JA: A simple weight decay can improve generalization. In Advances in neural information processing systems 4: 950-957, 1992.

40. Moody JE: The effective number of parameters: An analysis of generalization and regularization in nonlinear learning systems. In: Advances in Neural Information Processing Systems. Moody JE, Hanson SJ and Lippmann RP (eds). Morgan Kaufmann Publishers Inc., San Francisco, CA, pp847-854, 1992.

41. Youden WJ: Index for rating diagnostic tests. Cancer 3: 32-35, 1950.

42. Cohen J: A coefficient of agreement for nominal scales. Educ Psychol Meas 20: 37-46, 1960.

43. McHugh ML: Interrater reliability: The kappa statistic. Biochem Med (Zagreb) 22: 276-282, 2012.

44. Poljak M, Kovanda A, Kocjan BJ, Seme K, Jancar N and Vrtacnik-Bokal E: The abbott RealTime high risk HPV test: Comparative evaluation of analytical specificity and clinical sensitivity for cervical carcinoma and CIN 3 lesions with the Hybrid Capture 2 HPV DNA test. Acta Dermatovenerol Alp Pannonica Adriat 18: 94-103, 2009.

45. Tjalma WA, Fiander A, Reich O, Powell N, Nowakowski AM, Kirschner B, Koiss R, O'Leary J, Joura EA, Rosenlund M, et al: Differences in human papillomavirus type distribution in high-grade cervical intraepithelial neoplasia and invasive cervical cancer in Europe. Int J Cancer 132: 854-867, 2013.

46. De Sanjose S, Quint WG, Alemany L, Geraets DT, Klaustermeier JE, Lloveras B, Tous S, Felix A, Bravo LE, Shin HR, et al: Human papillomavirus genotype attribution in invasive cervical cancer: A retrospective cross-sectional worldwide study. Lancet Oncol 11: 1048-1056, 2010.

47. Lee SH, Vigliotti JS, Vigliotti VS and Jones W: From human papillomavirus (HPV) detection to cervical cancer prevention in clinical practice. Cancers (Basel) 6: 2072-2099, 2014. 
48. Miyagi Y, Fujiwara K, Oda T, Miyake T and Coleman RL: Development of new method for the prediction of clinical trial results using compressive sensing of artificial intelligence. J Biostat Biometric App 3: 203, 2018.

49. Abbod MF, Catto JW, Linkens DA and Hamdy FC: Application of artificial intelligence to the management of urological cancer. J Urol 178: 1150-1156, 2007.

50. Litjens G, Sánchez CI, Timofeeva N, Hermsen M, Nagtegaal I, Kovacs I, Hulsbergen-Van De Kaa C, Bult P, Van Ginneken B and van der Laak J: Deep learning as a tool for increased accuracy and efficiency of histopathological diagnosis. Sci Rep 6: 26286, 2016.

51. Khosravi P, Kazemi E, Zhan Q, Toschi M, Malmsten JE, Hickman C, Meseguer M, Rosenwaks Z, Elemento O, Zaninovic $\mathrm{N}$ and Hajirasouliha I: Robust automated assessment of human blastocyst quality using deep learning. BioRxiv: 394882, 2018.

52. Miyagi Y, Habara T, Hirata R and Hayashi N: Feasibility of deep learning for predicting live birth from a blastocyst image in patients classified by age. Reprod Med Biol 18: 190-203, 2019

53. Miyagi Y, Habara T, Hirata R and Hayashi N: Feasibility of artificial intelligence for predicting live birth without aneuploidy from a blastocyst image. Reprod Med Biol 18: 204-211, 2019.

54. Simões PW, Izumi NB, Casagrande RS, Venson R, Veronezi CD, Moretti GP, da Rocha EL, Cechinel C, Ceretta LB, Comunello E, et al: Classification of images acquired with colposcopy using artificial neural networks. Cancer Inform 13: $119-124,2014$.

55. Sato M, Horie K, Hara A, Miyamoto Y, Kurihara K, Tomio K and Yokota H: Application of deep learning to the classification of images from colposcopy. Oncol Lett 15: 3518-3523, 2018.

56. Ortiz A, Munilla J, Gorriz JM and Ramirez J: Ensembles of deep learning architectures for the early diagnosis of the Alzheimer's disease. Int J Neural Syst 26: 1650025, 2016.

57. Gil D, Johnsson M, Chamizo JMG, Paya AS and Fernandez DR: Application of artificial neural networks in the diagnosis of urological disfunctions. Expert Syst Appl 36: 5754-5760, 2009.

58. Olczak J, Fahlberg N, Maki A, Razavian AS, Jilert A, Stark A, Sköldenberg $\mathrm{O}$ and Gordon M: Artificial intelligence for analyzing orthopedic trauma radiographs. Acta Orthop 88: 581-586, 2017.
59. Sideri M, Garutti P, Costa S, Cristiani P, Schincaglia P, Sassoli de Bianchi P, Naldoni C and Bucchi L: Accuracy of colposcopically directed biopsy: Results from an online quality assurance programme for colposcopy in a population-based cervical screening setting in Italy. Biomed Res Int 2015: 614035, 2015.

60. Sideri M, Spolti N, Spinaci L, Sanvito F, Ribaldone R, Surico N and Bucchi L: Interobserver variability of colposcopic interpretations and consistency with final histologic results. J Lower Genital Tract Dis 8: 212-216, 2004.

61. Massad LS, Jeronimo J, Katki HA and Schiffman M; National Institutes of Health/American Society for Colposcopy and Cervical Pathology Research Group: The accuracy of colposcopic grading for detection of high-grade cervical intraepithelial neoplasia. J Lower Genital Tract Dis 13: 137-144, 2009.

62. LeCun Y, Haffner P, Bottou L and Bengio Y: Object recognition with gradient-based learning. Shape, contour and grouping in computer vision. Lecture Notes in Computer Science. Springer, Berlin, Heidelberg 1681: 319-345, 1999.

63. He K, Zhang X, Ren S and Sun J: Deep residual learning for image recognition. Proceedings of the IEEE conference on computer vision and pattern recognition, pp770-778, 2016.

64. Hu J, Shen L and Sun G: Squeeze-and-excitation networks. Proceedings of the IEEE conference on computer vision and pattern recognition, pp7132-7141, 2018.

65. Kudva V, Prasad K and Guruvare S: Automation of detection of cervical cancer using convolutional neural networks. Crit Rev Biomed Eng 46: 135-145, 2018.

66. Esteva A, Kuprel B, Novoa RA, Ko J, Swetter SM, Blau HM and Thrun S: Dermatologist-level classification of skin cancer with deep neural networks. Nature 542: 115-118, 2017.

This work is licensed under a Creative Commons Attribution-NonCommercial-NoDerivatives 4.0 International (CC BY-NC-ND 4.0) License. 